\title{
Evaluation of new Forcefield technology: Reducing platelet adhesion and cell coverage of pyrolytic carbon surfaces
}

\author{
Mark S. Slaughter, MD, ${ }^{\mathrm{a}}$ Brian Pederson, BS, ${ }^{\mathrm{c}}$ Joel D. Graham, MS, ${ }^{\mathrm{b}}$ Michael A. Sobieski, RN, CCP, ${ }^{\mathrm{a}}$ and \\ Steven C. Koenig, $\mathrm{PhD}^{\mathrm{a}, \mathrm{b}}$
}

\begin{abstract}
Introduction: Platelet adhesion and activation are a significant source of clinical complications. Preventing foreign surface-platelet interaction may improve biocompatibility of implantable medical devices. This study evaluated efficacy of novel technique for electrically modifying surface of conductive biomaterial and attaching blood components to prevent thrombogenesis. Specifically, this new surface modification technology, Forcefield (ATS Medical, Inc, Minneapolis, Minn), was designed to prevent platelet adhesion on pyrolytic carbon. A modulated low-voltage current is directly applied to pyrolytic carbon surfaces to stimulate adherence of a layer of charged proteins from circulating blood components that is resistive to platelet deposition.
\end{abstract}

\begin{abstract}
Methods: Feasibility of Forcefield technology was tested in line with cardiopulmonary bypass circuit in patients undergoing standard cardiac surgery $(\mathrm{n}=6)$. Forcefield treatment was applied to segment of pyrolytic carbon with 15 minutes $(n=3)$ and 30 minutes $(n=3)$ of electrically stimulated exposure time, and resulting segments were compared with untreated pyrolytic carbon segment. Platelet adhesion confluence was then quantified by scanning electron microscopy.

Results: Confluence of the Forcefield-treated pyrolytic carbon segments $(3.3 \% \pm 2.2 \%)$ was significantly reduced relative to untreated pyrolytic carbon control segments $(81.7 \% \pm 24 \%, P<.001)$. There were no discernible differences in cell confluence with Forcefield-treated segments as function of exposure time (15 or 30 minutes).

Conclusions: Forcefield technology may enable modification of pyrolytic carbon surfaces to prevent platelet adhesion and thrombogenesis of implanted medical devices, including heart valves, stents, catheters, and ventricular assist devices, and may eliminate the need for anticoagulant and antiplatelet therapies. (J Thorac Cardiovasc Surg 2011;142:921-5)
\end{abstract}

Biocompatibility of implantable medical devices has long been a concern for clinicians because of the morbidity associated with their use. This is especially true for those biomaterials in contact with blood, such as artificial heart valves, which currently carry a significant risk of thromboembolic events. Pyrolytic carbon (PYC) has been one of the most successful and widely used biomaterials by the medical device industry. PYC is considered an excellent biomaterial for use in medical devices ever since its original creation for use in nuclear reactors. ${ }^{1}$ Today, $\mathrm{PYC}$ remains the most popular biomaterial for implanted medical devices, as evidenced by its use in more than 4 million heart valves produced annually in more than 25 different design configurations. Despite the

\footnotetext{
From the Division of Thoracic and Cardiovascular Surgery, ${ }^{\mathrm{a}}$ Department of Surgery, and the Bioengineering Department, ${ }^{\mathrm{b}}$ University of Louisville, Louisville, Ky; and ATS Medical, Inc, ${ }^{\mathrm{c}}$ Minneapolis, Minn.

Disclosures: Authors have nothing to disclose with regard to commercial support.

Forcefield is a trademark of ATS Medical, Inc, Minneapolis, Minn.

Received for publication Oct 28, 2010; revisions received Dec 7, 2010; accepted for publication Jan 7, 2011; available ahead of print Feb 17, 2011

Address for reprints: Mark S. Slaughter, MD, Professor, Chief Division of Cardiothoracic Surgery, Director of Heart Transplant and Mechanical Assist Device Program, University of Louisville, Louisville, KY (E-mail: mark.slaughter@ louisville.edu).

$0022-5223 / \$ 36.00$

Copyright (C) 2011 by The American Association for Thoracic Surgery

doi:10.1016/j.jtcvs.2011.01.012
}

prevalence of PYC use, the requirement for chronic anticoagulation therapy remains a significant lifetime risk factor. Warfarin is generally administered and often supplemented with aspirin or dipyridamole to inhibit platelet activation, which is a contraindication for those patients who cannot tolerate long-term anticoagulation treatment. ${ }^{2}$

Ideally, biomaterial surfaces would have hydrophobic and hydrophilic properties, thereby attracting specific proteins to increase biocompatibility while repelling other blood components to prevent adhesion. Blood components such as albumin, the most abundant protein present in blood plasma, possess "passive" antithrombogenic and antibacterial properties. ${ }^{3,4}$ It has been reported that albumin is rapidly absorbed into the PYC biomaterial and that it inhibits platelet adhesion. ${ }^{5-7}$ Conversely, recent studies indicated a greater activation of platelets by exposure to PYC. ${ }^{8,9}$ To overcome the limitations of untreated PYC and take advantage of its potential antithrombogenic properties when albumin has been surface-bound, ATS Medical has developed a novel surface modification technique that results in a molecular layer of charged proteins and lipids bound to the surface of the PYC patented as Forcefield technology (ATS Medical, Inc, Minneapolis, Minn). In the Forcefield application process, a modulated low-voltage signal is applied directly to the PYC material while it is in contact with circulating blood. 


\section{Abbreviations and Acronyms \\ $\mathrm{CPB}=$ cardiopulmonary bypass \\ $\mathrm{PYC}=$ pyrolytic carbon}

It is hypothesized that this process of electrically stimulating the PYC surface will attract blood proteins and lipids to create a platelet-passive surface, producing an antithrombogenic layer. To test this hypothesis, Forcefield-treated PYC tubular segments were tested in a cardiopulmonary bypass (CPB) circuit during an arrested cardiovascular procedure.

\section{MATERIALS AND METHODS \\ Patients}

A single-center pilot study of the Forcefield technology was completed with 6 subjects ( 6 men; mean age, 65 years) who were undergoing elective coronary artery bypass grafting and required CPB support. None of the patients had any preoperative hematologic comorbidities, and their renal, hepatic, pulmonary, and neurologic functions were normal. All patients had preoperative platelet counts within the reference range $\left(181.2 \times 10^{6}\right.$ mean count).The institutional review board of Advocate Christ Medical Center (Oak Lawn, Ill) approved the research protocol, and informed consent was obtained from each patient before surgery.

\section{Design}

Untreated (control) and Forcefield-activated (treatment) segments of PYC (3.1 cm length, $1.0 \mathrm{~cm}$ inner diameter) were tested simultaneously. The sterilized test section was integrated in line with the CPB circuit proximal to the oxygenator, filtration system, and arterial return, which enabled the perfusionist to direct blood flow through the test section by closing or opening tubing clamps upstream and downstream of the test section (Figure 1). The electrical current used to generate the Forcefield was applied to the PYC test segment (treatment) by a function generator with the positive charged electrode (red) connected to the PYC segment and return electrode (black) connected to the titanium tube segment. The untreated (control) PYC segment was placed between the Forcefieldactivated PYC and titanium tube segments and was unaffected by the applied current (no charge applied or induced).

\section{Protocol}

In all 6 patients, $\mathrm{CPB}$ was initiated and blood cooled to $30^{\circ} \mathrm{C}$ in preparation for coronary artery bypass grafting. After cold blood cardioplegic arrest had been achieved, an electrical current was applied to the PYC Forcefield segment by applying an electropositive, offset-pulsed squarewave current at $17 \mathrm{~Hz}$ with a duty cycle of $41.6 \%$ (25 ms on, $60 \mathrm{~ms}$ off). The Forcefield treatment was applied for 15 minutes ( 3 patients) or 30 minutes ( 3 patients). The current applied to the Forcefield-treated PYC segment and titanium electrodes was quantified at the start of CPB perfusion and continuously monitored by oscilloscope during the Forcefield activation period. The electrical current applied to the PYC test segment was discontinued after the allotted Forcefield treatment time (15 or 30 minutes). CPB blood flow through the untreated (control) and Forcefield-treated (treatment) PYC test segments continued for duration of CPB support, with time of exposure to blood flow recorded.

\begin{abstract}
Analysis
Immediately after the discontinuation of $\mathrm{CPB}$, control and Forcefieldtreated PYC test segments were removed, rinsed in saline solution, and photographed with a digital camera. Each PYC test segment was fixed in glutaraldehyde and dehydrated in a series of alcohol baths for scanning electron microscopic analysis. PYC test segments were then cut longitudinally with a diamond saw to enable access to the interior surface. Scanning electron microscopic photos were taken at $25 \times, 200 \times$, and $1000 \times$ magnification. Scanning electron microscopic surface photos $(1000 \times)$ were used to quantify platelet-cell confluence. Adobe PhotoShop CS2 software (Adobe Systems Incorporated, San Jose, Calif) was used to quantify the area and percentage of cell involvement on the surface of each PYC test segment. ${ }^{10}$ Total pixel counts were calculated with the rectangular marquee tool. Confluence area was determined for each segment from the pixel number of cell confluence and the pixel number of the whole segment. The percentage surface area was calculated by dividing the confluence pixel number by the whole area pixel count.
\end{abstract}

\section{RESULTS}

No adverse events associated with this pilot study were observed, and there were no postoperative complications. All 6 patients who participated in this study were discharged from the hospital after a normal recovery period. Mean CPB time was 63 minutes, with a mean aortic crossclamp time of 43 minutes. In 5 of the 6 patients, the lowest temperature obtained during cardioplegic arrest was $30^{\circ} \mathrm{C}$. In 1 case of total circulatory arrest, the patient was cooled to $18^{\circ} \mathrm{C}$. The mean pulsed electrical current applied to the Forcefield-treated PYC test segment was $2.6 \mathrm{~mA}$, which was unaffected by $60-\mathrm{Hz}$ interference, noise, or distortion typically associated with operating room monitoring devices.

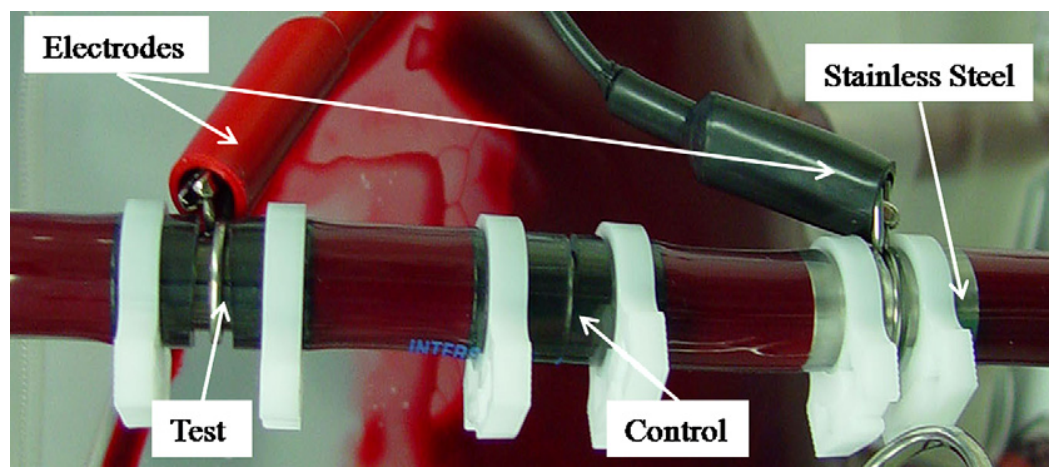

FIGURE 1. Forcefield test system consisted of treated test unit, which comprised section of stainless steel tubing and 2 sections of pyrolytic carbon tubing, $2.5 \mathrm{~cm}$ long; electronic circuit board and electrodes connected to oscilloscope; and function generator. 
TABLE 1. Summary of experimental parameters for comparison of untreated (control) and Forcefield-treated (test) pyrolytic carbon segments in line with cardiopulmonary bypass circuit for 6 patients during coronary artery bypass grafting

\begin{tabular}{lcccccc}
\hline & \multicolumn{3}{c}{ Contact time (min) } & & \multicolumn{2}{c}{ Confluence (\%) } \\
\cline { 2 - 3 } Patient & $\begin{array}{c}\text { Applied } \\
\text { current }\end{array}$ & $\begin{array}{c}\text { Post } \\
\text { current }\end{array}$ & $\begin{array}{c}\text { Total } \\
\text { contact }\end{array}$ & & $\begin{array}{c}\text { Control } \\
\text { PYC }\end{array}$ & $\begin{array}{c}\text { Forcefield } \\
\text { PYC }\end{array}$ \\
\hline 1 & 15 & 27 & 42 & 90 & 2.6 \\
2 & 30 & 38 & 68 & & 100 & 2.1 \\
3 & 15 & 62 & 77 & & 59 & 4.3 \\
4 & 30 & 46 & 76 & & 97 & 2.3 \\
5 & 15 & 44 & 59 & & 99 & 1.0 \\
6 & 30 & 62 & 92 & & 45 & 7.2 \\
\hline
\end{tabular}

Total contact time of pyrolytic carbon segments with circulating blood while in line with cardiopulmonary bypass varied patient to patient but was equivalent for control and Forcefield-treated segments for each patient. The percentage confluences for control and test pyrolytic carbon segments were quantified by scanning electron microscopy. PYC, Pyrolytic carbon.

Activation currents, contact times between PYC test segments and CPB circuit blood flow, and confluence results for the 6 patients are summarized in Table 1. Total contact time between PYC test segments and CPB circuit blood flow, which included a 15- or 30-minute Forcefield activation period, ranged from 42 to 92 minutes (average $69 \pm$ 17 minutes). The platelet confluences of the PYC control segments $(81.7 \% \pm 24 \%)$ were significantly greater $(P<.01)$ than those of the Forcefield-treated PYC segments $(3.3 \% \pm 2.2 \%)$, as shown in Figure 2.There were no statistically discernible differences in platelet confluence associated with Forcefield activation time.

Platelet confluence of the control (Figure 3,A) and treated (Figure 3, B) PYC surface sections looked distinctly different. The surface of the control section appeared to have a white, irregular accumulation of cells, whereas the test surface had a clean, polished appearance. The scanning electron microscopic analysis (Figure 4) confirmed the difference. The control surface (Figure 4, A) contained platelets throughout, whereas the treated surface (Figure $4, B$ ) had few areas of platelet coverage.

\section{DISCUSSION}

In this acute clinical study, Forcefield definitively inhibited platelet adhesion on treated test sections of PYC relative to untreated control PYC sections in a CPB circuit. These preliminary results demonstrate the potential for enhanced biocompatibility of Forcefield-treated PYC surfaces by reducing platelet adhesion and subsequent potential for thrombosis. In addition, because Forcefield treatment is localized to the biomaterial surface, there are no systemic alterations in the coagulation system.

Platelet confluence was notably decreased on the treated PYC test sections relative to the untreated control PYC sections. The specific mechanisms of action are unknown and continue to be investigated. Early in vitro results suggest that the electrically stimulated PYC attracts inherently charged blood components, such as albumin, fibrinogen, and phospholipids in a rapid and complete manner to create a platelet-passive surface, which is consistent with previous observations. ${ }^{8}$ Other studies have shown an anticoagulant effect of circulating albumin absorbed onto artificial surfaces that results from increased resistance to platelet adhesion. ${ }^{9,11,12}$ Still others have shown that a pulsating electric field can encourage platelet activation. ${ }^{13}$ With Forcefield, the applied electric current appears to cause albumin to be adsorbed rapidly. These formed layers may prevent subsequent platelet activation, adherence, and thrombosis.

The Forcefield technology has been previously tested in vitro to determine the optimal frequency, voltage, and current specifications for treating PYC surfaces (proprietary information) and in vivo in acute swine model experiments (unpublished pilot data). The PYC surfaces from these feasibility studies were extensively analyzed by gel

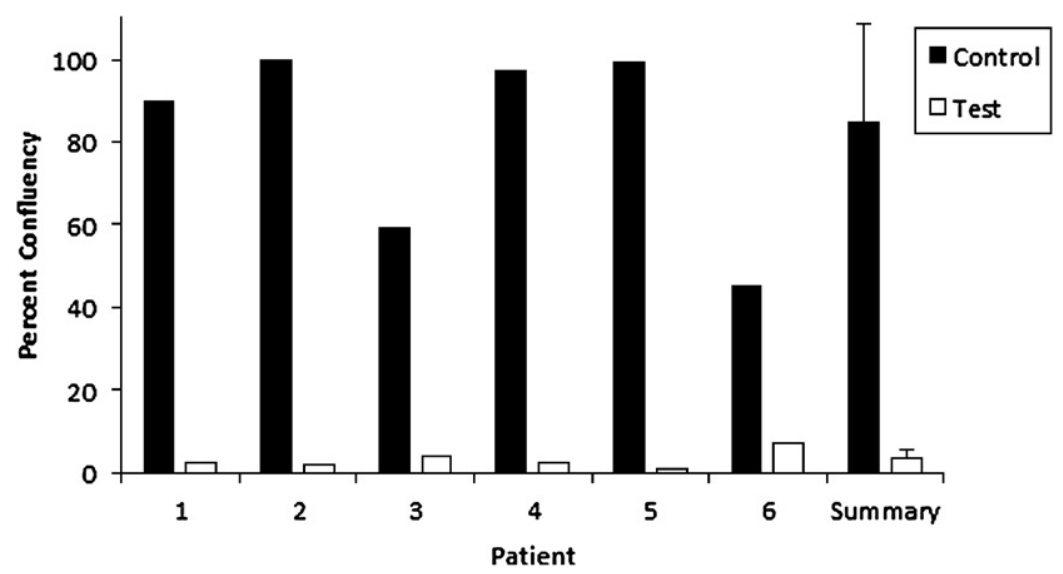

FIGURE 2. Platelet confluences on untreated section of pyrolytic carbon (Control) and treated section of pyrolytic carbon (Test) for 6 patients in study. Platelet confluence on control section ranged from $59 \%$ to $100 \%$ (mean $81.7 \% \pm 24 \%$ ); confluence on test section ranged from $1 \%$ to $7.2 \%$ (mean $3.3 \% \pm$ $2.2 \%$ ). 

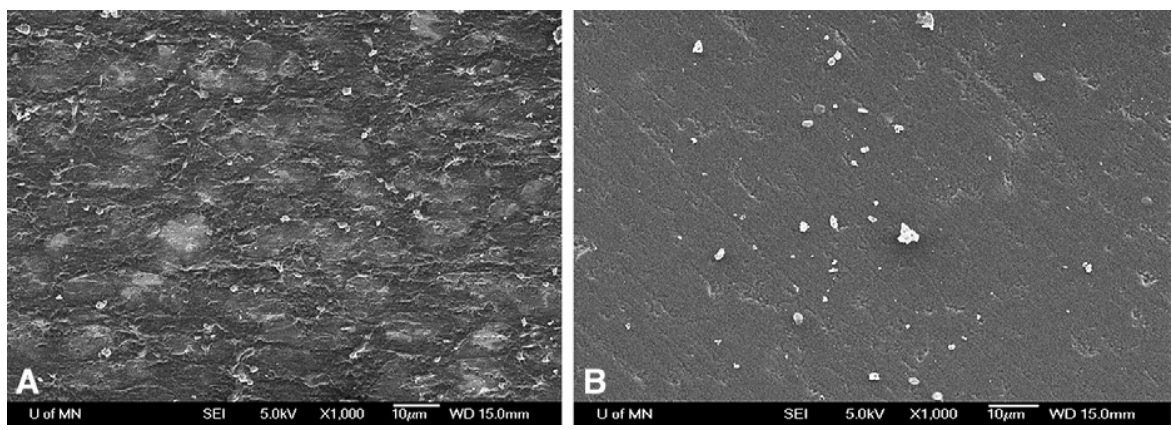

FIGURE 3. A, Appearance of untreated section of pyrolytic carbon (control). B, Appearance of treated section of pyrolytic carbon (test).

electrophoresis for preliminary surface protein assay analyses. These initial analyses showed a significant difference in surface content with respect to blood components on the Forcefield-treated, blood-perfused surfaces (Figure 5). The preliminary results suggest a difference in surface content with respect to the adherent blood components on the Forcefield-treated PYC surfaces. It is therefore hypothesized that albumin provides a stable charge on biomaterial surface, thus preventing electric charge-related platelet interactions as well as alteration of the fibrinogen receptors, which may inhibit the further binding of fibrinogen to the surface. ${ }^{14}$

PYC is an electrically conductive biomaterial that is ideal for surface charge modification and stimulation with an external power source. The electric current used in this study is similar both in current density and in morphology to that used for most implantable pulsed systems, such as standard cardiac pacemakers. We did not observe any adverse events in the patients participating in this study. Although we recognize the limitations of our small number of patients, we do not believe that Forcefield treatment will be associated with untoward effects. Important questions remain regarding the application of this technology, such as whether Forcefield treatment can be applied to other common implant biomaterials, how long the antithrombotic effect of Forcefield treatment will last, and how best to treat surfaces of various medical devices at implantation.

Current postoperative management of mechanical prosthetic valves includes the use of anticoagulation and antiplatelet therapies to minimize the risk of thrombosis and thromboembolic sequelae. These treatment options have risks associated with their long-term use. ${ }^{15}$ Therefore any therapy that has the potential to reduce the need for or amount of anticoagulation or antiplatelet therapies has value and merits further investigation. Possible applications for this biomaterial modification include the surfaces of many implantable intravascular devices, such as stents, mechanical heart valves, and ventricular assist devices, because patients with these devices often cannot tolerate anticoagulant and antiplatelet therapies. This technology could also potentially improve the biocompatibility of dialysis systems, oxygenators, and catheters. Although the preliminary acute clinical study data are promising, the Forcefield-treated PYC segments were exposed to blood for less than 2 hours. Additional testing to identify protective mechanisms, evaluate long-term durability, and validate efficacy are warranted.
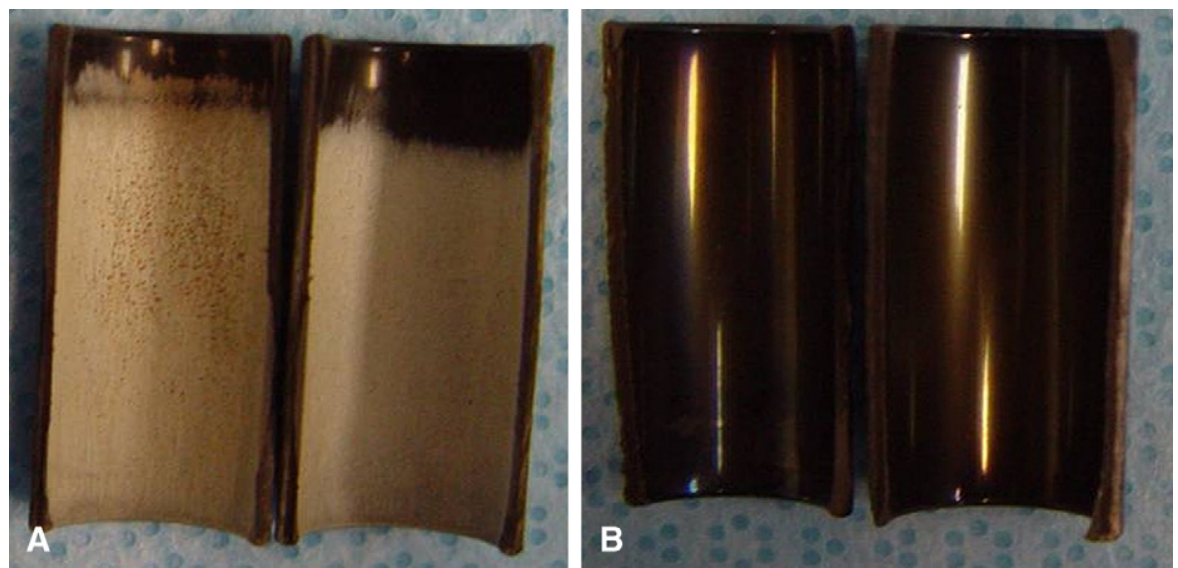

FIGURE 4. Scanning electron microscopy (original magnification 1000×). A, Appearance of untreated section of pyrolytic carbon (control). B, Appearance of treated section of pyrolytic carbon (test). 


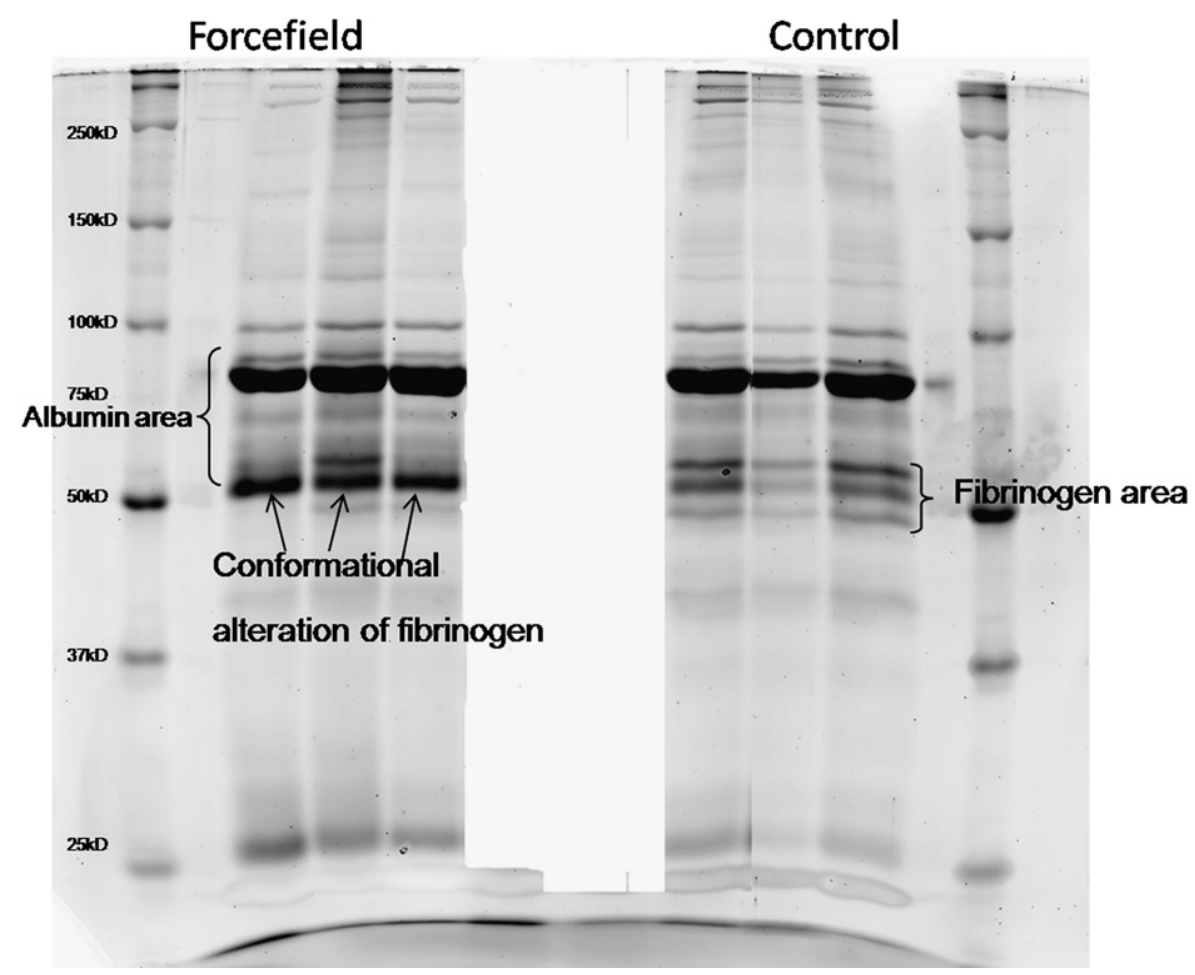

FIGURE 5. Gel electrophoresis analyses of untreated and Forcefield-treated test sections of pyrolytic carbon after exposure to human blood. Dark bands between 50 and $75 \mathrm{kD}$ indicate presence of albumin. It is hypothesized that albumin stabilizes charges on materials, preventing electric charge-related platelet interactions. At $50 \mathrm{kD}$, stimulation indicates possible change in composition of fibrinogen.

\section{References}

1. Hetherington VJ, Kawalec JS, Bhattacharyya B. Enhancement of the fixation of pyrolytic carbon implants by using atomic oxygen texturing. J Foot Ankle Surg. 2004;43:16-9.

2. Shishehbor MH, Goel SS, Kapadia SR, Bhatt DL, Kelly P, Raymond RE, et al. Long-term impact of drug-eluting stents versus bare-metal stents on all-cause mortality. J Am Coll Cardiol. 2008;52:1041-8.

3. Varbella F, Gagnor A, Tomassini F, Infantino V, Conte MR. Immediate and longterm results of treatment of complex lesions of the left anterior descending coronary artery involving a large diagonal branch with drug-eluting stents. J Cardiovasc Med (Hagerstown). 2008;9:1088-94.

4. Zapanta CM, Griffith JW, Hess GD, Doxtater BJ, Khalapyan T, Pae WE, et al. Microtextured materials for circulatory support devices: preliminary studies. ASAIO J. 2006;52:17-23.

5. Gott VL, Alejo DE, Cameron DE. Mechanical heart valves: 50 years of evolution. Ann Thorac Surg. 2003;76:S2230-9.

6. Chinn JA, Phillips RE, Lew KR, Horbett TA. Tenacious binding of fibrinogen and albumin to pyrolite carbon and biomer. J Colloid Interface Sci. 1996;184: $11-9$.

7. Goodman SL, Tweden KS, Albrecht RM. Platelet interaction with pyrolytic carbon heart valve leaflets. J Biomed Mater Res. 1996;32:249-58.
8. Goodman SL, Scranton VL, Brendzel AM. Platelet responses to silicon-alloyed pyrolytic carbons. J Biomed Mater Res A. 2007;83:64-9.

9. Mantero S, Piuri D, Montevecchi FM, Vesentini S, Ganazzoli F, Raffaini G. Albumin adsorption onto pyrolytic carbon: a molecular mechanics approach. J Biomed Mater Res. 2002;59:329-39.

10. Smith RN, Brook AH, Elcock C. The quantification of dental plaque using an image analysis system: reliability and validation. J Clin Periodontol. 2001;28:1158-62.

11. Houska M, Brynda E, Solovyev A, Broucková A, Krízová P, Vanícková M, et al. Hemocompatible albumin-heparin coatings prepared by the layer-by-layer technique. The effect of layer ordering on thrombin inhibition and platelet adhesion. J Biomed Mater Res A. 2008;86:769-78.

12. Tsai CC, Huo HH, Kulkami P, Eberhart RC. Biocompatible coatings with high albumin affinity. ASAIO Trans. 1990;36:M307-10.

13. Zhang J, Blackmore PF, Hargrave BY, Xiao S, Beebe SJ, Schoenbach KH Nanosecond pulse electric field (nanopulse): a novel non-ligand agonist for platelet activation. Arch Biochem Biophys. 2008;471:240-8.

14. Chin JA, Phillips RE Jr, Lew KR, Horbett TA. Tenacious binding of fibrinogen and albumin to pyrolyte carbon and biomer. J Colloid Interface Sci. 1996;184:11-9.

15. Chan KE, Lazarus JM, Thadhani R, Hakim RM. Warfarin use associates with increased risk for stroke in hemodialysis patients with atrial fibrillation. $J \mathrm{Am}$ Soc Nephrol. 2009;20:2223-33. 